\title{
The English Lexicon Project
}

\author{
David A. Balota And Melvin J. YaP \\ Washington University, St. Louis, Missouri \\ Michael J. Cortese \\ College of Charleston, Charleston, South Carolina \\ KEITH A. HUTCHISON \\ Montana State University, Bozeman, Montana \\ Brett KessLer AND BJoRn LofTIS \\ Washington University, St. Louis, Missouri \\ JAMES H. NEELY \\ University at Albany, State University of New York, Albany, New York \\ Douglas L. Nelson \\ University of South Florida, Tampa, Florida \\ GREG B. SIMPSON \\ University of Kansas, Lawrence, Kansas \\ AND \\ RebecCa Treiman \\ Washington University, St. Louis, Missouri
}

\begin{abstract}
The English Lexicon Project is a multiuniversity effort to provide a standardized behavioral and descriptive data set for 40,481 words and 40,481 nonwords. It is available via the Internet at elexicon.wustl.edu. Data from 816 participants across six universities were collected in a lexical decision task (approximately 3400 responses per participant), and data from 444 participants were collected in a speeded naming task (approximately 2500 responses per participant). The present paper describes the motivation for this project, the methods used to collect the data, and the search engine that affords access to the behavioral measures and descriptive lexical statistics for these stimuli.
\end{abstract}

One could easily argue that the most commonly studied stimulus set in experimental psychology involves English words. The study of the memory and reading of words has been central to research since Cattell (1886). Words are well-described units that provide the link between perception and meaning, and so have been critical to developments in computational modeling (e.g., McClelland \& Rumelhart, 1981), neuroimaging (e.g., Petersen, Fox, Posner, Mintun, \& Raichle, 1989), and conceptions of attention and automaticity (e.g., Neely, 1977; Stroop, 1935), among many other research areas.

Given the importance of words as a stimulus set, one might assume that there are relatively straightforward ways to study lexical processing, and that there is a wellconstrained set of findings to which one can appeal in building models of word processing. Although there has been considerable progress in understanding how people process words, there are some clear gaps in the available literature. This paper describes the English Lexicon Project (ELP), which provides a behavioral database for over 40,000 words and nonwords that will help fill some of these gaps. The present description will focus on visual word recognition, although, as described below, the current database has relevance for other aspects of word processing, such as memory and speech production. Before describing the ELP, we will briefly describe the behavioral measures in the database, the limitations in our current knowledge, and how this database will help address these limitations.

\section{LEXICAL DECISIONS AND NAMING AS THE BEHAVIORAL TARGETS}

Although there are multiple ways to measure lexical processing (e.g., eye-fixation data, probability of iden-

D.A. Balota,dbalota@artsci.wustl.edu 
tifying degraded stimuli, semantic verification), most work on isolated visual word recognition has employed two relatively simple tasks, lexical decision and speeded naming. In the lexical decision task (LDT), participants are presented with a string of letters (either a word or a nonword, e.g., FLIRP), and are asked to press one button if the string is a word and another button if the string is a nonword. In the speeded naming task, participants see a visual word (or sometimes a nonword), and are asked to name the word aloud as quickly and as accurately as possible. Although researchers have clearly demonstrated task-specific effects in the LDT and naming task (e.g., Balota \& Chumbley, 1984, 1985; Keefe \& Neely, 1990; Neely, Keefe, \& Ross, 1989), these two tasks are still the major driving force in research on isolated word recognition and have been the gold standard in developing computational models of lexical processing (see Coltheart, Rastle, Perry, Langdon, \& Ziegler, 2001; Grainger \& Jacobs, 1996; Plaut, McClelland, Seidenberg, \& Patterson, 1996; Seidenberg \& McClelland, 1989; Zorzi, Houghton, \& Butterworth, 1998). The ELP includes measures of both lexical decision and naming performance.

\section{WHAT ARE THE LIMITATIONS IN CURRENT KNOWLEDGE?}

Researchers investigating the processes involved in visual word recognition typically employ factorial designs in which item variables (e.g., word frequency, spelling-tosound regularity, neighborhood density, syntactic class) are "manipulated" on a relatively small set of items (typically 10 to 20 items per condition) and the effects of these variables on the speed and accuracy of lexical decision and/or naming performance are measured. In the vast majority of these studies, a mean is calculated for each participant across items (or for each item across participants in some studies) and then entered into an analysis of variance, and the effects of "factors" are measured. Although this approach has been fruitful in identifying a number of important variables that modulate speeded lexical decision and naming performance, it is also clear that this approach has some potential limitations.

First, it is difficult to select a set of items that only vary on one dimension, while controlling the myriad of variables that have been identified in the literature (see Cutler, 1981). For simplicity, consider the influence of spelling-to-sound correspondences, such as the fact that the word PINT is not pronounced according to common spelling-to-sound principles, whereas HINT is consistent with such principles. The influence of irregular spellingto-sound correspondence will depend on a number of factors such as the frequency of the target word, the number and frequency of neighbors with similar spelling-to-sound correspondences (friends), the number and frequency of neighbors with different spelling-to-sound correspondences (enemies), and probably a host of other variables (see, e.g., Jared, McRae, \& Seidenberg, 1990). A single set of 10 to 20 stimuli used in a given experiment may indeed show idiosyncratic influences of the multitude of uncontrolled variables that limits the generalizability of the targeted variable to other sets of items.

Second, one may be concerned that implicit knowledge by the researcher could bias the selection of words for an experiment. For example, Forster (2000) has shown that researchers in word recognition can predict very well which of two relatively well-matched stimuli would yield faster lexical decision latencies. Hence, it appears that researchers possess knowledge of lexical processing that could implicitly or explicitly play a role in the selection of stimuli to test hypotheses in a given experiment.

Third, list contexts (e.g., the other items in a list) can influence how a variable affects performance in a word recognition experiment. For example, Andrews (1997), in a review of the orthographic neighborhood size literature, has argued that the inconsistencies in the lexical decision task data are largely driven by differences in stimulus list environments (e.g., legal, FLIRP, vs. illegal, FLPRI, nonword foils) across experiments. Thus, list contexts can modulate the presence, magnitude, and even direction of experimental effects in the literature.

Fourth, the vast majority of lexical processing research has been dedicated to single-syllable words, and hence, many experiments have exclusively used lists that only include such words. The major computational models that have been developed by Coltheart et al. (2001), Plaut et al. (1996), and Seidenberg and McClelland (1989) have all exclusively dealt with monosyllabic words (but see Ans, Carbonnel, \& Valdois, 1998, for an example of a multisyllabic computational model). Many interesting issues arise with multisyllabic words, such as the role of syllable structure, stress patterning, morphological structure, type of length effects (e.g., orthographic vs. phonological vs. syllabic length), among many others. It is even possible that single-syllable lists may produce a list context effect that may directly modulate the results.

\section{THE ENGLISH LEXICON PROJECT}

Given the richness of the English lexicon, along with the problems noted above, it is appropriate to expand the horizons of experimental work addressing lexical processing beyond standard factorial experiments that are based on a relatively limited set of monosyllabic stimuli. The ELP is such an attempt. The ELP is a large database of descriptive and behavioral data, along with a search engine that affords access to this database. The ELP involves a multiuniversity collaborative effort to provide a large database of naming and lexical decision latencies across 1,260 participants for 40,481 words and nonwords. The goal of this project is to provide a database that is easily accessible for researchers from diverse backgrounds and interests that can at least minimize the concerns raised above with current approaches to lexical processing.

A few researchers have gone beyond traditional factorial studies to provide analyses of large databases. Such megastudies provide estimates of word recognition performance for a large set of words (Balota \& Spieler, 1998; Besner \& Bourassa, 1995; Kessler, Treiman, \& 


\section{Naming}

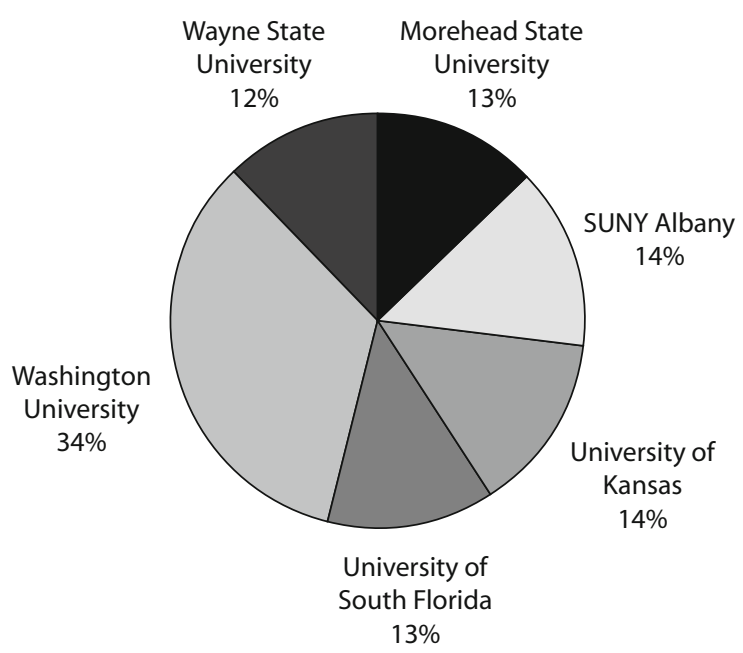

Lexical Decision

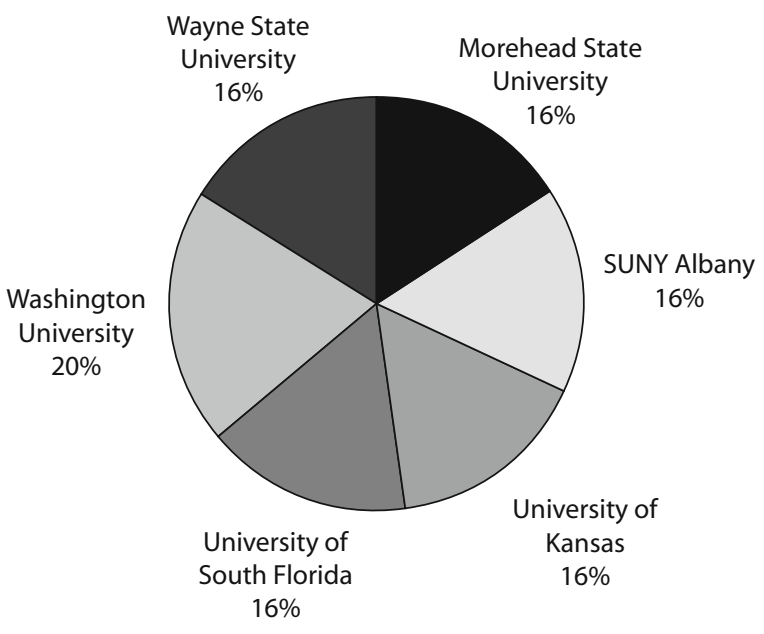

Figure 1. Proportion of participants from each institution.

Mullennix, 2002; Spieler \& Balota, 1997; Treiman, Mullennix, Bijeljac-Babic, \& Richmond-Welty, 1995). For example, Treiman et al., using multiple regression and speeded naming latencies as a dependent variable, examined the effects of different subsyllabic units on naming performance. Similarly, by examining the lexical decision and naming latencies to 2,428 words (Balota \& Spieler, 1998; Spieler \& Balota, 1997), Balota, Cortese, SergentMarshall, Spieler, and Yap (2004) demonstrated that standard psycholinguistic variables (e.g., length, frequency, orthographic neighborhood size) were able to account for nearly half the variance in the behavioral data. However, such large-scale studies are relatively rare, tend to focus mostly on speeded naming performance, are not easily accessible to the research community, and are almost always based on single syllable words (see Chateau \& Jared, 2003, for an exception). The ELP is the logical extension to this body of work, and provides normative word recognition performance for 40,000 mono- and multisyllabic words, along with a search engine that affords access to a rich set of descriptive lexical characteristics. We will now turn to a presentation of the ELP, and later in the General Discussion section will present some of the many possible uses of this database.

\section{METHOD}

\section{Participants}

Participants were native English speakers recruited from research participant pools in the six testing institutions (see Figure 1 for a breakdown of the proportion of participants from each institution and Table 1 for descriptive statistics of participant demographics for the two tasks). The universities included private and public institutions across the Midwest, Northeast and Southeast regions of the United States, and at each institution a word recognition researcher directed the collection of data. Participants were either paid $\$ 25$ for participation in the two sessions or received credit for course requirements. Each participant participated in either the naming task or the lexical decision task. Participants took part in two different sessions that were conducted on different days, separated by no more than one week. At the end of the second testing sessions, participants also received the Morningness-Eveningness Questionnaire (a circadian rhythm questionnaire) (Horne \& Ostberg, 1976), the Shipley (1940) vocabulary test, and a general health questionnaire. Based on the consistency of the previous data sets that have been developed (e.g., Balota \& Spieler, 1998), we decided that an individual participant could produce stable data for approximately 2,500 (naming) to 3,500 stimuli (lexical decision).

\section{Apparatus}

The experimental software and testing apparatus were identical at each of the six testing sites. Stimuli were presented via a 19-in. Trinitron monitor (P991), which was controlled by a Pentium III PC. Stimuli were presented in uppercase in the standard QBASIC font in the 80 (column) $\times 30$ (row) mode. For the naming task, there were two microphones. One microphone (Sony condenser microphone, ECMMS907) was connected to a Sony DAT recorder (PCM-M1) for future offline analyses of the naming data. The second was a head-mounted Shure microphone (WH20QRT), which was connected to an in-housedeveloped electronic voice relay. This voicekey was modeled after the Gerbrands G1341T microphone (no longer commercially available) and had similar response characteristics as the Gerbrands voicekey.

Table 1

Means and Standard Deviations of Participant Demographics for the Speeded Naming and Lexical Decision Tasks

\begin{tabular}{|c|c|c|c|c|}
\hline & \multicolumn{2}{|c|}{$\begin{array}{l}\text { Speeded Naming } \\
\quad(n=444)\end{array}$} & \multicolumn{2}{|c|}{$\begin{array}{l}\text { Lexical Decision } \\
\quad(n=816)\end{array}$} \\
\hline & Mean & $S D$ & Mean & $S D$ \\
\hline Age & 23.51 & 9.31 & 22.86 & 6.85 \\
\hline Years of Education & 14.73 & 1.75 & 14.76 & 1.72 \\
\hline Shipley Vocabulary Age & 17.59 & 1.28 & 17.39 & 1.35 \\
\hline Mean RT & 735.06 & 106.69 & 787.86 & 164.83 \\
\hline Percent Errors & 8.41 & 4.77 & 14.40 & 6.90 \\
\hline *a. Uncertain of pronunciation & 3.81 & 2.90 & & \\
\hline *b. Mispronunciation & 2.91 & 2.12 & & \\
\hline${ }^{*}$ c. Voice key problem & 1.09 & 1.18 & & \\
\hline *d. Time out & 0.60 & 2.20 & & \\
\hline Percent Outliers & 4.90 & 2.96 & 3.57 & 3.74 \\
\hline
\end{tabular}

*These coding categories are only applicable to the speeded naming task, where participants coded their own responses. 


\section{Stimuli}

Because of the enormous potential size of the candidate set of words for this project, we were compelled to make decisions to limit the size of the master list. The master list of 40,481 words was based initially on the Kučera and Francis (1967) norms, and then was supplemented by the CELEX (Baayen, Piepenbrock, \& Gulikers, 1993) norms. The Kučera and Francis stimuli were initially screened so that stimuli that were clearly nonwords (probably due to typographical errors) or had nonalphabetic characters (e.g., $\mathrm{A} * * \$ R E$ ) were eliminated. Because the target set of participants in this study were undergraduates, we had three undergraduate research assistants at Washington University, under the direction of a graduate student and a faculty member, assist in the selection of the items. Words that were eliminated included obscenities, slurs, misspellings, and alternate (e.g., British English) spellings. Common multimorphemic words were included, but because of space constraints, we could not include all possible derivatives for a given stem. For example, JOYFUL and JOYFULLY were kept but UNJOYFULLY was removed. Proper names and contractions were included. After the master list was constructed, nonwords were created for the lexical decision task. Pronounceable nonwords were generated by changing one or two letters in a corresponding target word. The location of the letter change alternated across different words to include early, middle, and late positions.

\section{Creation of Sublists for Individual Participants}

Naming. Participants either received 2,530 or 2,531 items. For all 40,481 words to be sampled once, 16 participants ( 15 with 2,530 and 1 with 2,531 ) were necessary. To obtain 25 observations per word, 400 participants were necessary $(16 \times 25=400)$. In order to assign words to sublists, words in the master list $(40,481$ items) were first sorted alphabetically. To minimize the likelihood that an individual participant would name words with the same morphemic stem, the first 16 adjacent words in the master list were randomly assigned to 16 sublists. This process was repeated until there were no unassigned words in the master list. This process of assigning words from the master list to the 16 sublists was repeated 25 times, resulting in a sublist for each of the 400 participants to be tested. This process also insured that, for each group of 16 participants, no 2 participants named the same word. Practice and buffer items (see below) were taken from other participants in the group of 16 (i.e., Participant 1 received buffer items from Participant 2's test lists).

Lexical decision. A similar procedure was used to create sublists for the lexical decision task. For LDT, since there was a nonword for each word, the total number of items in the master list was 80,962 . For the LDT, 24 participants ( 17 with 3,374 and 7 with 3,372 ) were necessary for a single observation for all 80,962 items. Hence, 816 $(24 \times 34=816)$ participants afforded 34 observations per item in lexical decision performance. For the LDT, words were sorted alphabetically, and when words were assigned to the sublists, the corresponding nonword was also assigned to the same sublists. To minimize the number of times a participant would see words with the same root, 24 adjacent words in the master list were randomly assigned to 24 sublists.

\section{Procedures}

Naming task. Participants received 40 practice trials before each experimental session. The practice trials for a given participant were selected from a different counterbalanced list. Each session contained several blocks of 250 trials (the last block of the last session was either 280 or 281 trials). The first session consisted of 1,500 trials; the second session was either 1,030 or 1,031 trials. Each block of 250 trials was followed by a rest break. The break after practice trials was $15 \mathrm{sec}$ long. At 750 trials, the break was 3 min long. All other breaks (after each 250 trials) were 1 min long. During 3-minlong breaks, the computer screen instructed participants to "Please use this time to get a drink, stretch, or walk around." A countdown timer was shown at the bottom of the screen to inform participants how much time was remaining in the break.
During each break, the proportion of accurate trials and mean response latencies were presented for the 250 trials preceding the break. If the accuracy was $80 \%$ or greater, a message "Please maintain this level of accuracy" was presented. If the accuracy was less than $80 \%$, a message "Please increase your level of accuracy" was presented. If the mean response latency was less than $1,000 \mathrm{msec}$, a message "Please maintain this reaction time" was presented. If the mean response latency was greater than $1,000 \mathrm{msec}$, a message "Please decrease your response time" was presented. After the break, three auditory tones were presented to alert the participant. The participant was instructed to press B to begin the next block. A blank screen was presented $2.5 \mathrm{sec}$ after the keypress and before two buffer trials initiated the next test block.

The sequence of events during each naming trial was as follows: (a) three asterisks were presented at the center of the screen for $250 \mathrm{msec}$; (b) a $50-\mathrm{msec}$ tone was then presented indicating the onset of the next trial; (c) a 250-msec dark interval was presented; (d) the target word was presented centered at the same location the asterisks were presented; (e) the participant named the word; (f) the computer detected the voice onset; $(\mathrm{g})$ the word remained on the screen for an additional $250 \mathrm{msec}$ after voice onset; (h) the word was erased from the screen. If the response latency for the vocal response was less than $4,000 \mathrm{msec}$, a screen appeared asking the participant to manually code the accuracy of their response. The four choices were 1) correct pronunciation, 2) uncertain of pronunciation, 3) mispronunciation, and 4) microphone error. Participants were instructed beforehand regarding the importance and use of these coding options.

If the voice key detected a sound while the word was initially presented ( 0 -msec response latency), an error message and a warning tone was presented. The trial was reinitiated after a 2,500-msec delay. If no response was made within $4,000 \mathrm{msec}$, the item was removed from the screen and the words "Too slow" were presented for $500 \mathrm{msec}$. At the end of each trial, a blank screen was displayed during the 1,000-msec intertrial interval.

Lexical decision task. There were 40 practice trials before each experimental session. As in naming, each session contained several blocks of 250 trials (the last block of the last session was only 122 or 124 trials). The first session included 2,000 trials, whereas the second session included either 1,372 or 1,374 trials. The breaks were identical to the naming task, including the feedback concerning accuracy and response latency.

The sequence of events during each trial was the same as in the naming task, except that the participant pressed the "/" key for a word response and the " $z$ " key for a nonword response. If the participant responded before the letter string was presented, an error message on the screen and tone were presented and the trial was reinitiated after a 2,500-msec delay. If no response was made within $4,000 \mathrm{msec}$, the item was removed from the screen and the words "Too slow" were presented for $500 \mathrm{msec}$. If the response was inaccurate, the word "Incorrect!" appeared for $500 \mathrm{msec}$. If the participant pushed an invalid key, instructions about the key mapping appeared for $2,000 \mathrm{msec}$. At the end of the trial, a blank screen was displayed during the $1,000-\mathrm{msec}$ intertrial interval.

\section{DESCRIPTION OF LEXICAL CHARACTERISTICS AND BEHAVIORAL MEASURES}

In this section, we describe the information that is available at the website at elexicon.wustl.edu. Descriptive statistics for words (see Table 2) and nonwords (Table 3) are also included. Interested users are encouraged to access the website to explore the range of variables available.

In order to ensure that extreme response latencies did not disproportionately influence the item mean correct reaction time for the naming and lexical decision tasks, we used a two-step outlier identification procedure: First, any response latencies faster than $200 \mathrm{msec}$ or slower 
Table 2

Descriptive and Behavioral Data for the Words Used in the English Lexicon Project

\begin{tabular}{|c|c|c|c|c|}
\hline & Min & Max & $M$ & $S D$ \\
\hline \multicolumn{5}{|l|}{ General Fields } \\
\hline Length & 1 & 21 & 8.00 & 2.46 \\
\hline $\mathrm{K} \& \mathrm{~F}$ frequency & 1 & 69,971 & 29.73 & 557.64 \\
\hline HAL frequency & 0 & $23,099,033$ & $10,778.67$ & $192,226.37$ \\
\hline Log HAL frequency & 0 & 17 & 6.16 & 2.40 \\
\hline \multicolumn{5}{|l|}{ Orthographic Neighborhood } \\
\hline Type orthographic neighborhood size & 0 & 25 & 1.29 & 2.73 \\
\hline Token orthographic neighborhood size & 0 & 16 & 7.02 & 1.88 \\
\hline Type neighborhood frequency (greater) & 0 & 21 & 1.69 & 2.32 \\
\hline Token neighborhood frequency (greater) & 1 & 16 & 8.42 & 1.93 \\
\hline Type neighborhood frequency (less) & 0 & 23 & 1.69 & 2.41 \\
\hline Token neighborhood frequency (less) & 0 & 16 & 5.89 & 1.74 \\
\hline \multicolumn{5}{|l|}{ Bigram Frequency } \\
\hline Summed bigram frequency & 11 & 59,803 & $14,313.09$ & $7,848.76$ \\
\hline Average bigram frequency & 5.5 & 6,910 & $1,974.56$ & 729.99 \\
\hline Summed bigram frequency by position & 1 & 6,603 & $2,149.21$ & $1,070.14$ \\
\hline \multicolumn{5}{|l|}{ Phonological Characteristics } \\
\hline Pronunciation & NA & & & \\
\hline Number of phonemes & 1 & 17 & 6.70 & 2.28 \\
\hline Number of syllables & 1 & 8 & 2.54 & 1.10 \\
\hline \multicolumn{5}{|l|}{ Morphological Characteristics } \\
\hline Morpheme parse-letters & NA & & & \\
\hline Morpheme parse-phonemes & NA & & & \\
\hline Number of morphemes & 1 & 7 & 2.11 & .86 \\
\hline \multicolumn{5}{|l|}{ Behavioral Results: Lexical Decision } \\
\hline Mean LDT latency (raw) & & & 784.07 & 134.93 \\
\hline Mean LDT latency (standardized) & & & .00 & .46 \\
\hline Standard deviation of LDT latencies & & & 277.51 & 92.39 \\
\hline Number of observations & & & 27 & 6.92 \\
\hline Mean accuracy & & & .84 & .20 \\
\hline \multicolumn{5}{|l|}{ Behavioral Results: Naming } \\
\hline Mean naming latency (raw) & & & 722.58 & 109.00 \\
\hline Mean naming latency (standardized) & & & -.04 & .46 \\
\hline Standard deviation of naming latencies & & & 178.47 & 69.30 \\
\hline Number of observations & & & 24.00 & 4.04 \\
\hline Mean accuracy & & & .93 & .11 \\
\hline
\end{tabular}

than 3,000 msec were identified as outliers. Second, for the remaining correct RTs, a mean and standard deviation were calculated, and any RT that was less than $3 S D$ s below the mean for that participant or greater than $3 S D$ s above the mean for that participant was also identified as being an outlier. The mean item latencies for naming and lexical decision performance, along with the $z$-score estimates, described below, are based on the remaining correct observations across the participants who received that particular item. Also, as described below, one can access the individual participant level data if one wishes to utilize an alternative screening procedure.

\section{CHARACTERISTICS AVAILABLE FROM SUBMITTING AND GENERATING LISTS OF ITEMS}

As described further below, the user may submit a list of items to generate characteristics for that list of items or have the website gen-

Table 3

Descriptive and Behavioral Data for the Nonwords Used in the English Lexicon Project

\begin{tabular}{lcrrr}
\hline & Min & Max & \multicolumn{1}{c}{$M$} & \multicolumn{1}{c}{$S D$} \\
\hline $\begin{array}{l}\text { General Fields } \\
\quad \text { Length }\end{array}$ & 1 & 21 & 8.00 & 2.46 \\
$\begin{array}{l}\text { Orthographic Neighborhood } \\
\quad \text { yype orthographic neighborhood size }\end{array}$ & 0 & 24 & 1.78 & 2.22 \\
Bigram Frequency & & & & \\
$\quad$ Summed bigram frequency & 11 & 56,657 & $13,562.11$ & $7,537.63$ \\
$\quad$ Average bigram frequency & 10.5 & 5,197 & $1,867.73$ & 714.78 \\
$\quad$ Summed bigram frequency by position & 0 & 6,985 & $1,936.96$ & 952.82 \\
Behavioral Results: Lexical Decision & & & & \\
$\quad$ Mean LDT latency (raw) & & & 855.78 & 113.75 \\
Mean LDT latency (standardized) & & & -.24 & .36 \\
Standard deviation of LDT latencies & & & 299.02 & 85.43 \\
$\quad$ Number of observations & & & 28.72 & 4.87 \\
Mean accuracy & & & .88 & .13 \\
\hline
\end{tabular}


erate a list of items with specific constraints. Within both of these commands, the following set of variables can easily be accessed.

\section{General Fields}

Length is the number of letters in the word.

Freq_KF refers to the Kučera and Francis (1967) frequency norms. These norms are derived from a corpus of 1,014,000 words drawn from a variety of American English texts. Although these norms are available in the data set, we strongly encourage users to use the HAL norms (described below) or some other estimate of word frequency since it has been repeatedly demonstrated that the Kučera and Francis norms are relatively poor estimates of raw frequency, most likely because of its small corpus (see, for e.g., Balota et al., 2004; Zevin \& Seidenberg, 2002).

Freq_HAL refers to the Hyperspace Analogue to Language (HAL) frequency norms (Lund \& Burgess, 1996), based on the HAL corpus, which consists of approximately 131 million words gathered across 3,000 Usenet newsgroups during February 1995.

Log_Freq_HAL refers to log-transformed HAL frequency norms.

\section{Orthographic Neighborhood Characteristics}

Ortho_N is the number of words that can be obtained by changing one letter while preserving the identity and positions of the other letters (i.e., Coltheart's N; Coltheart, Davelaar, Jonasson, \& Besner, 1977). For example, the ELP returns the following orthographic neighbors of CAT: OAT, COT, VAT, CAB, MAT, CAM, BAT, RAT, CAD, HAT, CAP, PAT, FAT, SAT, EAT, CAR, CUT, CAN.

Freq_N is the mean log of HAL frequencies of the orthographic neighbors of a particular word.

Freq_Greater is the number of orthographic neighbors of an item that are more frequent than that item based on the HAL frequencies.

Freq_G_Mean is the mean log of HAL frequencies of the orthographic neighbors for an item that are more frequent than that item.

Freq_Less is the number of orthographic neighbors for an item that are less frequent than that item based on the HAL frequencies.

Freq_L_Mean is the mean log of HAL frequencies of orthographic neighbors for an item that are less frequent than that item.

Freq_Rel is the log HAL frequency of an item divided by the mean log HAL frequency of its orthographic neighbors.

Neighborhood Button permits a user to display the actual orthographic neighbors of a submitted word or nonword.

\section{Bigram Frequency Characteristics}

BG_Sum refers to summed bigram frequency, where bigram is defined as a sequence of two letters. The summed bigram frequency of a letter string (e.g., DOG) is the sum of the frequencies of its successive bigrams (i.e., DO \& OG).

$B G \_$Mean refers to average bigram frequency, which is the summed bigram frequency divided by the number of successive bigrams.

BG_Freq_By_Pos refers to summed bigram frequencies by position, and is based on bigram frequencies that are sensitive to positions within words. Position-sensitive bigram counts take into account the letter positions where a bigram occurs. For example, the bigram frequency for DO in DOG counts DO bigrams only when they appear in the first two positions of a word.

\section{Phonological Characteristics}

Pronunciation provides phonological transcriptions for words, and is largely based on the Unisyn Lexicon developed by the Centre for Speech Technology Research at the University of Edinburgh (Centre for Speech Technology Research, University of Edinburgh, n.d.).

Number of phonemes provides the phoneme count for a word. For example, CAT / kæt/ has three phonemes.

Number of syllables provides the syllable count for a word. For example, CAT / kæt/ has one syllable.

\section{Morphological Characteristics}

Morpheme Parse - Letters parses words into morphemes (units of meaning), using letter segments (e.g., CATS is parsed as CAT-S).
Morpheme Parse-Phonemes parses words into morphemes, using phoneme segments (e.g., CATS is parsed as kæt-s).

Number of Morphemes provides the morpheme count for a word. For example, CATS has two morphemes, /CAT/ + /S/.

\section{Part of Speech}

Part of Speech provides the word's part of speech, and shows how the word is used. Parts of speech include verbs, nouns, adjectives, and prepositions.

\section{BEHAVIORAL RESULTS}

\section{Lexical Decision}

$I \_M e a n \_R T$ is the mean lexical decision latency (in $\mathrm{msec}$ ) for a particular word across participants.

I_Zscore provides the standardized mean lexical decision latency for each word. Each participant's raw lexical decision latencies are standardized using a $z$-score transformation, and the mean $z$-score for all participants presented with a particular word is then computed. This metric allows the lexical decision performance for different words to be directly compared, with more negative $z$ scores denoting shorter latencies. Because there is considerable variability across participants in overall response latency and each participant only receives a subset of the stimuli, the standardized item score is the most reliable measure, minimizing the influence of a participant's processing speed and variability (see Faust, Balota, Spieler, \& Ferraro, 1999).

$I \_S D$ is the standard deviation of lexical decision latencies for a word across participants.

$O b s$ is the number of observations available for a word.

I_Mean_Accuracy is the proportion of accurate responses for a particular word, excluding errors and outliers.

\section{Naming}

$I \_N M G \_M e a n \_R T$ is the mean naming latency (in msec) for a particular word across participants.

I_NMG_Zscore provides the standardized mean naming latency for a word. This metric allows the naming performance for different words to be directly compared, with more negative $z$-scores denoting shorter latencies (see above).

$I \_N M G \_S D$ is the standard deviation of naming latencies for a word across participants.

I_NMG_Obs is the number of observations available for a word.

I_NMG_Mean_Accuracy is the proportion of accurate responses for a particular word, excluding errors and outliers.

\section{Lexical Decision to Nonwords}

$N W I \_M e a n \_R T$ is the mean lexical decision latency (in msec) for a particular nonword across participants.

NWI_Zscore is the standardized lexical decision latency for a nonword. This metric allows the lexical decision performance for different nonwords to be directly compared, with more negative $z$-scores denoting shorter latencies.

NWI_SD is the standard deviation of lexical decision latencies for a nonword across participants. 


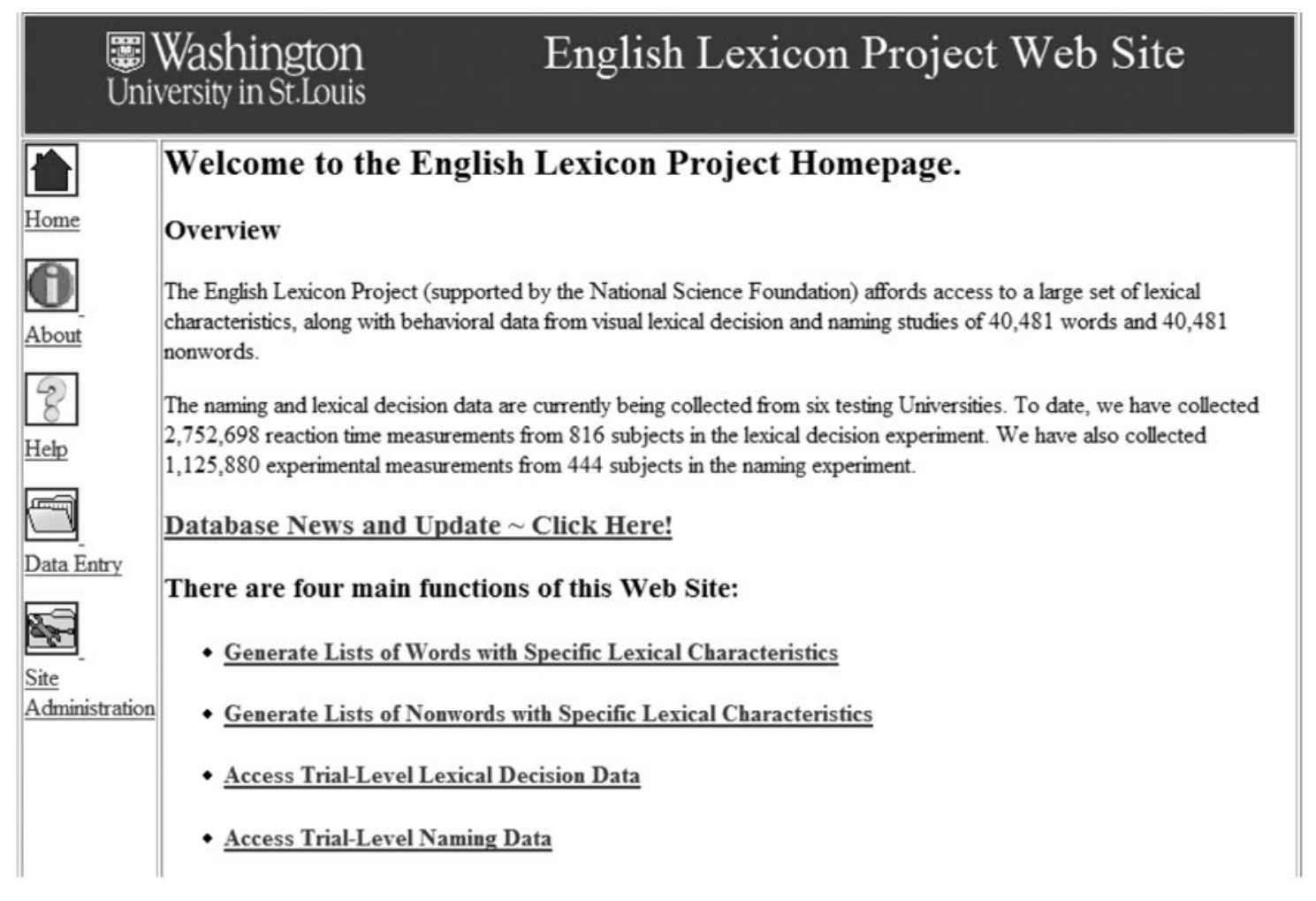

Figure 2. The English Lexicon Project homepage.

Please select the fields and any contraints to be used in the query:

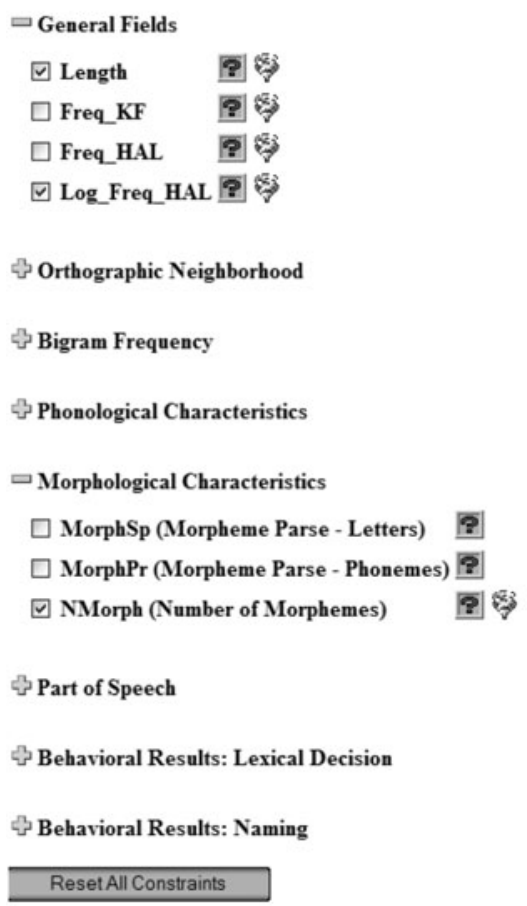

Figure 3. Interface for generating word lists with specific characteristics. 


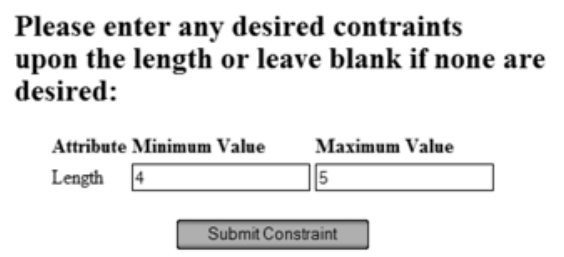

Figure 4. Placing constraints on the length variable.

$O b s$ is the number of observations available for a nonword.

NWI_Mean_Accuracy is the proportion of accurate responses for a particular nonword, excluding errors and outliers.

\section{INDIVIDUAL PARTICIPANT DATA}

In addition to accessing item information, the database also affords the opportunity to access individual participant data. The following describes the list of parameters used for such selection that are available for the lexical decision and naming data.

Sub_ID refers to participants' unique ID.

Trial refers to the position a trial appears within an experimental block.

Type refers to the lexicality of a trial, where word $=1$ and nonword $=0$, only applicable for lexical decision data.

D_Accuracy refers to trial accuracy, where accurate $=$ 1 and error $=0$.

$D \_R T$ refers to trial response time (in $\mathrm{msec}$ ).

D_word shows the specific item presented on a trial.

Outlier indicates whether the response time for a trial is extreme or not, where outlier $=1$ and nonoutlier $=0$ (see above description of outlier screening procedure).

$D \_Z s c o r e$ refers to $z$-standardized response times, with more negative $z$-scores denoting shorter latencies.

\section{USING THE ELP QUERIES}

In this section we will describe how to use the search engine available at the ELP website. There are of course multiple configurations of the requests. Here we provide specific examples, but direct exploration of the website and search engine is encouraged.

As noted, the ELP has four basic functions:
(1) Generate a list of stimuli with specific characteristics

(2) Submit a list of stimuli to obtain lexical characteristics and behavioral performance measures (i.e., naming and lexical decision) for these stimuli

(3) Submit a list of arbitrary nonword stimuli to obtain lexical characteristics

(4) Provide access to individual participants' data

\section{(1) Generate a List of Stimuli With Specific Characteristics}

This function allows users to generate stimulus sets with prespecified parameters. Users can select the characteristics they are interested in, as well as place constraints on the range of values for these characteristics. For example, a user might be interested in obtaining a pool of words which are four to five letters in length, low frequency (e.g., $\log$ HAL frequency $\leq 4$ ), and monomorphemic. The instructions for doing this are provided below.

a. Go to the ELP homepage (elexicon.wustl.edu) (Figure 2), and click on 'Generate Lists of Words with Specific Lexical Characteristics’.

b. Click on 'Generate Lists of Items with Specific Lexical Characteristics'.

c. This brings the user to the next page (Figure 3), where the different lexical and behavioral categories are displayed. Clicking on the ' + ' icon expands a particular field. For example, clicking on 'General Fields' reveals the length and frequency measures. For our query, we will check 'Length', 'Log_Freq_ HAL', and 'NMorph'.

d. The icon to the right of the question mark is the constraint button, and allows users to set the range of values for their measure of interest. For example, to select words that are four to five letters long, click on the constraint button for 'Length'. This brings up a pop-up box (Figure 4), where 4 and 5 can be, respectively typed into the 'Minimum Value' and 'Maximum Value' boxes. Repeat this step for 'Log_Freq_ HAL' (Minimum Value $=0$, Maximum Value $=4$ ), and 'NMorph' (Minimum Value = 1, Maximum Value $=1$ ).
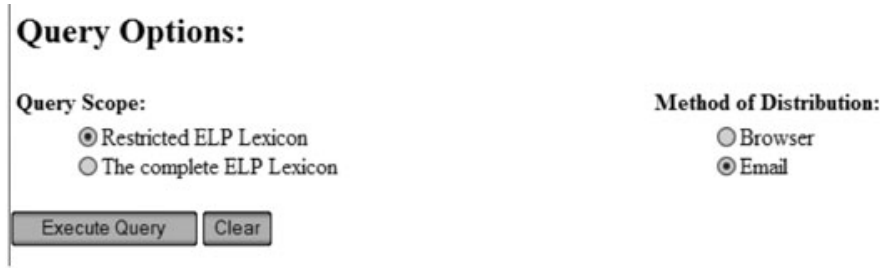

Figure 5. Options for method of distribution. 


\begin{tabular}{|c|c|c|c|c|c|c|}
\hline \multicolumn{7}{|c|}{$\mathbb{3}$ Microsoft Excel - I12979.csy } \\
\hline 잠 & File Edit & Yiew & ser & t Format & Iools $\quad$ & Dat. \\
\hline \multicolumn{7}{|c|}{ 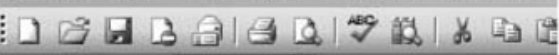 } \\
\hline \multicolumn{2}{|c|}{ G7 } & $\checkmark$ & $f_{2}$ & & & \\
\hline & A & B & & C & D & \\
\hline 1 & Word & Length & & og_Freq_ & NMorph & \\
\hline 2 & abash & & & 0 & & 1 \\
\hline 3 & wrack & & & 3.76 & & 1 \\
\hline 4 & yawl & & & 3.4 & & 1 \\
\hline 5 & yokel & & & 3.97 & & 1 \\
\hline 6 & whorl & & & 3.37 & & 1 \\
\hline 7 & ulna & & & 2.77 & & 1 \\
\hline 8 & true & & & 0 & & 1 \\
\hline 9 & thrum & & & 3.18 & & 1 \\
\hline 10 & tilth & & & 2.89 & & 1 \\
\hline 11 & tepee & & & 2.77 & & 1 \\
\hline 12 & tamp & & & 3.4 & & 1 \\
\hline 13 & swash & & & 3.56 & & 1 \\
\hline 14 & swum & & & 3.83 & & 1 \\
\hline 15 & strew & & 5 & 2.56 & & 1 \\
\hline
\end{tabular}

Figure 6. List of words with specific lexical characteristics.

e. At this point, the user has to respond to the two query options, 'Query Scope' and 'Method of Distribution' (Figure 5). 'Query Scope' is, by default, limited to the restricted ELP lexicon. Selecting the complete ELP lexicon yields orthographic neighborhood and bigram frequency measures that are computed using a larger 79,672 word corpus based on the HAL norms. In contrast, the restricted ELP lexicon computes these measures using the 40,481 word lexicon presented to the ELP participants. Turning to 'Method of Distribution', the user can choose to view the query output directly on the browser or to receive it as an e-mail attachment. It is usually better to ask for the output as an attachment via the e-mail option, given the large amount of information that is generated by the typical query.

f. Once these options are selected, click on the 'Execute Query' button (see Figure 5), and the website will ask for an e-mail address if the Method of Distribution is via e-mail. Output will be sent to the email address provided, in a comma-delimited file (Figure 6) that can be opened in any text editor or spreadsheet program. If the Method of Distribution is via the Browser, the results will be displayed immediately on the screen.

g. The procedure for generating a list of nonwords with specific lexical characteristics is very similar. From the homepage, click on 'Generate Lists of Nonwords with Specific Lexical Characteristics', followed by 'Search for nonwords matching your criteria'. The remaining steps are conceptually very similar to the instructions described above. Note that only a subset of lexical characteristics (length, orthographic neighborhood, and bigram frequency) are available for nonwords.
(2) Submit a List of Stimuli To Obtain Lexical Characteristics and Behavioral Performance Measures for These Stimuli

This function allows users to submit lists of words and to obtain lexical characteristics and behavioral performance measures for these items. For example, a user may wish to obtain length, frequency counts, and naming latencies for a list of words.

a. Starting from the ELP homepage, click on 'Generate Lists of Words with Specific Lexical Characteristics'.

b. Click on 'Submit Lists of Items to generate Lexical Characteristics'.

c. The list of words can be either submitted as an ASCII text file (one word on each line), or be pasted or typed directly into a box on the browser. To submit a file, select 'Filename Containing List of Words' (Figure 7). To do the latter, select 'Free Text List of Words'.

d. Next, select the fields of interest. To obtain length and frequency counts for the submitted stimuli, check all four fields under 'General Fields' (Figure 8). In addition, check 'I_NMG_Mean_RT' under 'Behavioral Results: Naming'.

e. For 'Query Scope' and 'Method of Distribution', follow the instructions described in Step 1e.

f. Click on the 'Execute Query' button, which will either present a blank box to enter stimuli, or will ask for the path of the to-be-submitted text file, depending on the method of submission chosen in Step 2c.

g. The output can then be viewed directly on the browser or be received as an e-mail attachment, depending on the method of distribution selected in

\section{List Options:}

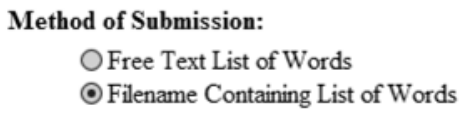

Figure 7. Options for submitting items.

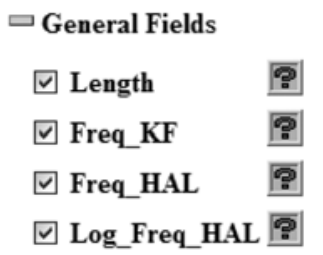

Figure 8. Selecting all the General Fields. 


\begin{tabular}{|c|c|c|c|c|c|c|c|}
\hline & A & B & L & $u$ & ヒ & $r$ & $\mathrm{G}$ \\
\hline 1 & Occurances & Word & Length & Freq_KF & Freq_HAL & Log_Freq_HAL & I_NMG_Mean_RT \\
\hline 2 & 1 & abash & 5 & NULL & 1 & 0 & $59 \overline{4} .43$ \\
\hline 3 & 1 & strew & 5 & NULL & 13 & 2.56 & 777.81 \\
\hline 4 & 1 & swash & 5 & NULL & 35 & 3.56 & 654.88 \\
\hline 5 & 1 & swum & 4 & 1 & 46 & 3.83 & 752.48 \\
\hline 6 & 1 & tamp & 4 & 1 & 30 & 3.4 & 665.24 \\
\hline 7 & 1 & tepee & 5 & NULL & 16 & 2.77 & 768.2 \\
\hline 8 & 1 & thrum & 5 & NULL & 24 & 3.18 & 690.53 \\
\hline 9 & 1 & tilth & 5 & 1 & 18 & 2.89 & 725 \\
\hline 10 & 1 & true & 4 & 231 & 0 & 0 & 699.79 \\
\hline 11 & 1 & ulna & 4 & NULL & 16 & 2.77 & 740 \\
\hline 12 & 1 & whorl & 5 & NULL & 29 & 3.37 & 788.33 \\
\hline 13 & 1 & wrack & 5 & 1 & 43 & 3.76 & 626.92 \\
\hline 14 & 1 & yawl & 4 & 1 & 30 & 3.4 & 651.68 \\
\hline 15 & 1 & yokel & 5 & 2 & 53 & 3.97 & 608.4 \\
\hline
\end{tabular}

Figure 9. Lexical and behavioral characteristics of submitted words.

Step 2e. For the latter option, note that two commadelimited text files will be sent as attachments. One file provides lexical and behavioral characteristics for individual items (Figure 9), while the other file provides summary statistics of the selected characteristics for the submitted items (Figure 10).

h. The procedure for submitting a list of nonwords to obtain specific lexical characteristics is very similar. From the homepage, click on 'Generate Lists of Nonwords with Specific Lexical Characteristics', followed by 'Submit lists of nonwords in the database and obtain lexical characteristics'. The remaining steps are conceptually very similar to the instructions described above. For this query, note that only a subset of lexical characteristics are available for nonwords, and that submitted nonwords have to be in the ELP nonword database in order to generate an output. For nonwords not represented in the database, see Function 3 (described below).

\section{(3) Submit a List of Arbitrary Nonword Stimuli To Obtain Lexical Characteristics}

This function allows users to obtain lexical characteristics for lists of nonwords, whether these words are represented in the database or not. For example, a user may wish to obtain orthographic neighborhood and bigram frequency characteristics for a set of nonwords.

a. Starting from the ELP homepage, click on 'Generate Lists of Nonwords with Specific Lexical Characteristics'.

b. Click on 'Submit arbitrary lists of nonwords in and generate lexical characteristics'. c. The list of nonwords can be either submitted as an ASCII text file (one word on each line), or be pasted directly into a box on the browser. To submit a file, select 'Filename Containing List of Words'. To do the latter, select 'Free Text List of Words'. For more details, follow the instructions described in Step 2c.

d. Next, select the fields of interest. For our example, we will select all the available lexical characteristics (Figure 11).

e. For 'Query Scope' and 'Method of Distribution', follow the instructions described in Step 1e.

f. Click on the 'Execute Query' button, which will either present a blank box to enter stimuli, or will ask for the path of the to-be-submitted text file, depending on the method of submission chosen in Step 3c. Once these options are selected, click on the 'Execute Query' button, and the website will ask for an e-mail address, if Method of Distribution is e-mail. Output (see Figures 12 and 13) will be sent to the e-mail address provided, in a comma-delimited file that can be opened in any text editor or spreadsheet program. Again, if Method of Distribution is browser, the data will be immediately displayed on screen.

\section{(4) Provide Access to Individual Participants' Data}

This function provides access to the raw lexical decision and naming latencies.

a. Starting from the ELP homepage, click on 'Access Lexical Decision and Naming Data'.

\begin{tabular}{l|r|r|r|r|r|r|r|r|r} 
& \multicolumn{1}{|c|}{ A } & B & C & D & E & F & G & H \\
\hline 1 & Total_Count & Distinct_Count & Imvalid_Count & Mean_Length & Mean_Freq_KF & Mean_Freq_HAL_Mean_Log_Freq_HAL Mean_I_NMG_Mean_RT \\
\hline 2 & 14 & 14 & 0 & 4.64 & 34 & 25.29 & 2.82 & 695.98 \\
\hline
\end{tabular}

Figure 10. Summary statistics of submitted words. 


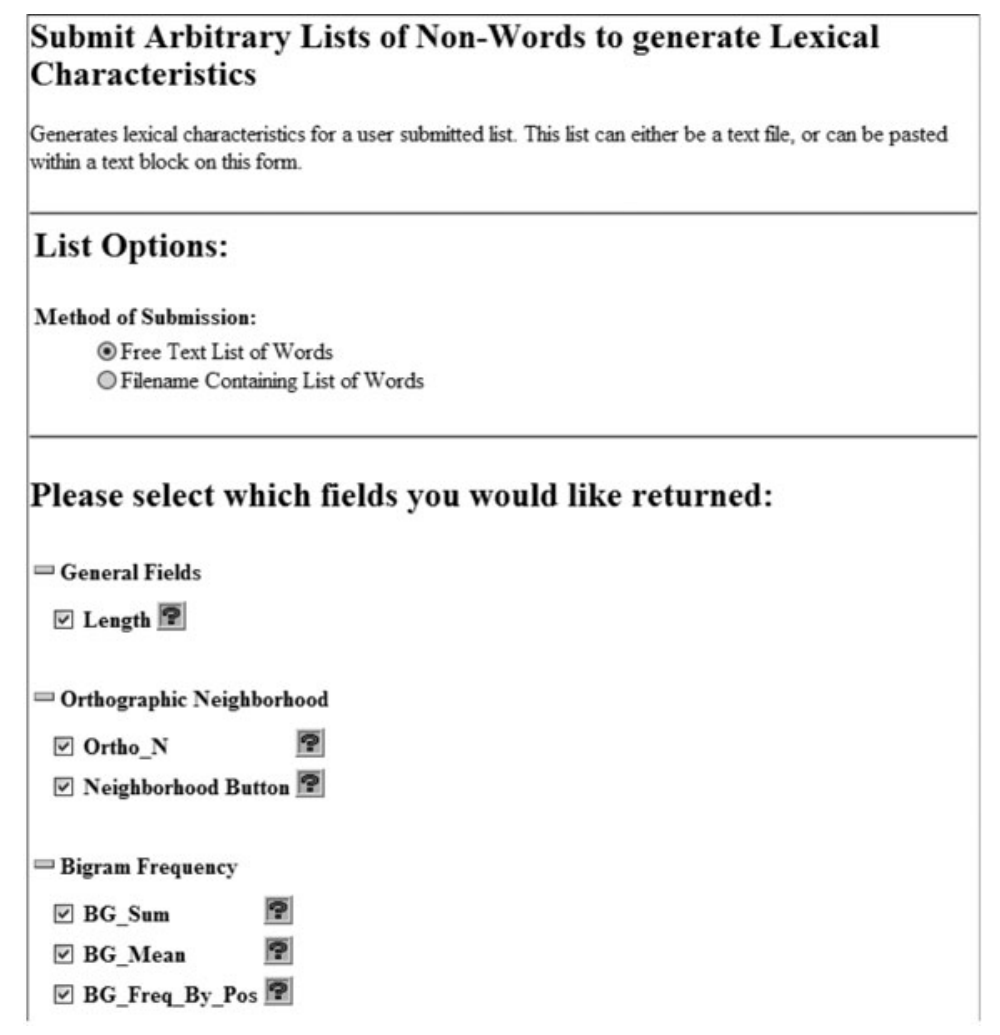

Figure 11. Interface for submitting novel nonwords.

b. Depending on whether the user is interested in retrieving lexical decision data or naming data, click on 'Access Trial-Level Lexical Decision Data' or 'Access Trial-Level Naming Data', respectively. In the following example, lexical decision trial data for the first five participants will be retrieved, with only accurate word trials selected.

c. The previous step brings the user to the page depicted in Figure 14. Clicking on the ' + ' icon expands General Fields. For our purposes, check all the options (descriptions provided below) available.

d. A number of constraints can be set by the user.

i. Sub_ID specifies the participants whose data are to be downloaded.

ii. Trial specifies the trials (e.g., first 100 trials) of interest iii. Type specifies lexicality (words $=1$; nonwords $=0$ ); only applicable to lexical decision

iv. D_Accuracy specifies trial accuracy (correct $=1$; error $=0$ )

v. $D \_R T$ specifies trial response times (in msec)

vi. D_word refers to the specific items presented on a trial

vii. Outlier specifies whether a trial is an outlier (outlier $=$ True; nonoutlier $=$ False $)$

viii. D_Zscore specifies $z$-transformed response times

d. Click on the constraint buttons for 'Sub_ID', enter 1 as the 'Minimum value' and 5 as the 'Maximum value' (Figure 15). This selects the first five participants.

\begin{tabular}{r|r|r|r|r|r|r|r|}
\hline & \multicolumn{1}{c|}{ A } & B & C & D & E & F & G \\
\hline 1 & Total_Count & Distinct_Count & Mean_Length & Mean_Ortho_N & Mean_BG_Sum Mean_BG_Mean Mean_BG_Freq_By_Pos \\
\hline 2 & 26 & 26 & 4.96 & 3.58 & 5774.69 & 1436.96 & 1171.04 \\
\hline
\end{tabular}

Figure 12. Summary statistics of submitted nonwords. 


\begin{tabular}{r|r|l|r|r|r|r|r|}
\hline & \multicolumn{1}{|c|}{ A } & B & \multicolumn{1}{c|}{ C } & \multicolumn{1}{c|}{ D } & \multicolumn{1}{c|}{ E } & \multicolumn{1}{c|}{ F } & \multicolumn{1}{c|}{ G } \\
\hline 1 & Occurances & Word & Length & Ortho_N & BG_Sum & BG_Mean & BG_Freq_By_P0S \\
\hline 2 & 1 LEMM & 4 & 0 & 4809 & 1603 & 731 \\
\hline 3 & 1 BOUD & 4 & 4 & 2796 & 932 & 947 \\
\hline 4 & 1 DRAS & 4 & 5 & 4958 & 1652.67 & 1308 \\
\hline 5 & 1 SOID & 4 & 6 & 1978 & 659.33 & 518 \\
\hline 6 & 1 FLOD & 4 & 8 & 2477 & 825.67 & 748 \\
\hline 7 & 1 DALT & 4 & 9 & 3969 & 1323 & 853 \\
\hline 8 & 1 LARN & 4 & 9 & 5457 & 1819 & 1433 \\
\hline 9 & 1 SOIN & 4 & 9 & 8027 & 2675.67 & 846 \\
\hline 10 & 1 SOUT & 4 & 14 & 3399 & 1133 & 958 \\
\hline 11 & 1 SPRUD & 5 & 0 & 3194 & 798.5 & 581 \\
\hline 12 & 1 HOOVE & 5 & 0 & 4562 & 1140.5 & 1170 \\
\hline 13 & 1 THRID & 5 & 0 & 5293 & 1323.25 & 1000 \\
\hline 14 & 1 POPLE & 5 & 0 & 6019 & 1504.75 & 1208 \\
\hline 15 & 1 SHOIR & 5 & 1 & 3648 & 912 & 928 \\
\hline
\end{tabular}

Figure 13. Characteristics of submitted nonwords.

e. Following this, for 'Type', enter 1 for both minimum and maximum values (selects only words). For 'D_Accuracy', enter 1 for both minimum and maximum values (selects only accurate responses).

f. Finally, to have the data sent to you, under query options, go to 'Method of Distribution' in the 'Query Options' box (Figure 16). The user selects whether the data are presented on the browser or sent as an e-mail attachment. Given the typically large size of the data files, the e-mail option is recommended.

\section{DISCUSSION}

In this paper, we have described the English Lexicon Project (ELP), a Web-based repository encompassing descriptive and behavioral measures for over 80,000 items, based on data collected from nearly 1,300 participants at six different universities. We have presented the methods used to collect the data, defined the variables included in the database, and illustrated how the search engine facilitates access to the ELP's many resources.

One reservation researchers might have about the ELP is that naming or making lexical decisions to 3,000 items

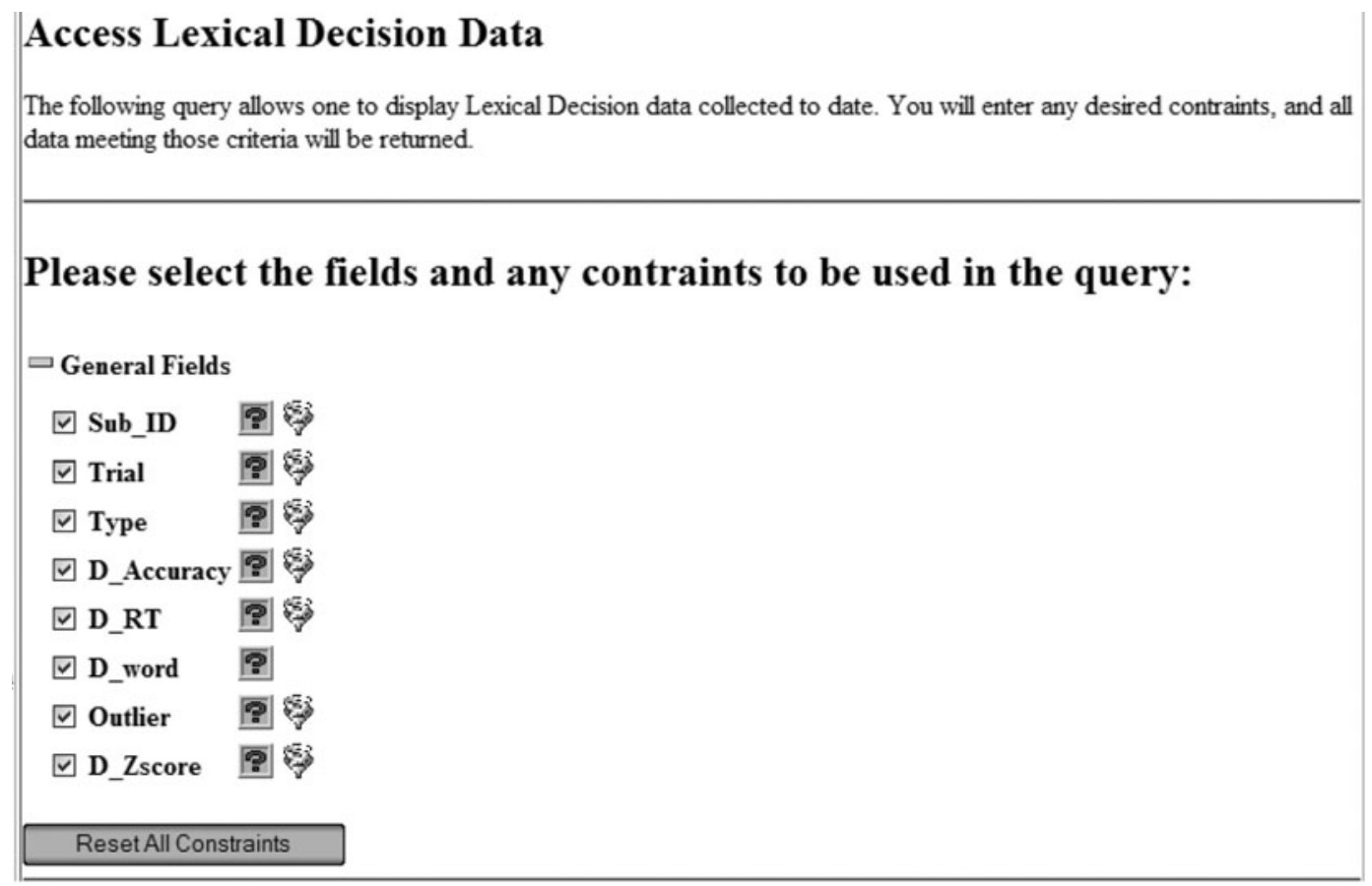

Figure 14. Interface for accessing individual trial-level data. 


\section{Please enter any desired contraints upon the SUB_ID or leave blank if none are desired:}

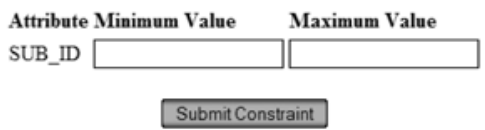

Figure 15. Placing constraints on the subject ID variable.

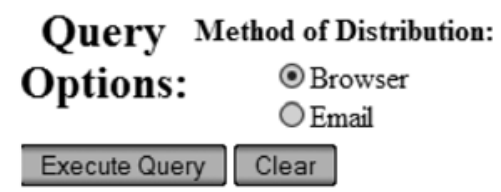

Figure 16. Options for method of distribution.

would produce variability due to fatigue or boredom. If this were the case, then one might worry that these data sets will be relatively noisy and have limited utility. Hence, traditional word recognition studies involving 100 to 200 words may produce a better reflection of normal lexical performance. There are a number of responses to this potential concern. First, Spieler and Balota (1997) demonstrated that four simple predictor variables (log frequency, neighborhood density, orthographic length, and phonological onset) accounted for $42 \%$ of the item level variance in a large-scale behavioral data set of naming performance. Thus, there is a considerable amount of predictable variance in these data sets. Second, although the estimates for proportion of variance accounted for fluctuate slightly across different large data sets, one observes the same pattern of predictive power in the data sets obtained by Seidenberg and Waters (1989), Spieler and Balota (1997), and Treiman et al. (1995). Finally, Balota and Spieler (1998) selected the same items from a large-scale study that were used in a standard factorial study conducted by Taraban and McClelland (1987, Experiment $1 \mathrm{~A})$. The results were remarkably similar to the pattern reported by Taraban and McClelland (see Balota \& Spieler, 1998, Figure 1).

\section{Potential Uses for the Website}

We envision this database being used in a number of different types of research endeavors. First, as noted, most computational models are based on single-syllable words, and the vast majority of studies of lexical processing have only included single-syllable words, and so this literature has produced a relatively restricted account of lexical processing. The present research affords a database to extend these models to multisyllabic (and multimorphemic) stimuli.

Second, these data will be important for researchers interested in targeting particular variables. In some cases, experiments might be replaced by accessing the database. As described above, the database can be easily accessed to provide naming and lexical decision data for any set of words and/or nonwords in the database. Because of the size of the database, one can resample different sets of items with particular characteristics from the database. In other situations, researchers may use the database as a preliminary step in developing further follow-up experiments to test the influence of a given variable. The advantage of the present database is that it will minimize the idiosyncratic effects that could arise because of specific selection of items or list contexts, described earlier. We believe that the standardization of this data set will be helpful in minimizing the inconsistencies that have arisen in the word recognition literature. One might be concerned that there may be a bias for researchers to search for items that actually produce an effect in the data. Of course, this is also a problem in the current word recognition literature. Ultimately, the peer review process will be responsible for evaluating the potential for this problem in any given paper.

Third, researchers from other disciplines outside of word recognition (e.g., memory, perception, neuroimaging, and neuropsychology) will be able to use this database to select items that are equated along a number of relevant dimensions. The ELP can generate word and nonword lists based

\begin{tabular}{|c|c|c|c|c|c|c|c|c|}
\hline & A & $B$ & C & D & $E$ & $\mathrm{~F}$ & G & $\mathrm{H}$ \\
\hline 1 & Sub_ID & Trial & Type & D_Accuracy & D_RT & D_word & Outlier & D_Zscore \\
\hline 2 & 3 & 5 & 1 & 1 & 721 & butting & 0 & -0.31935 \\
\hline 3 & 3 & 10 & 1 & 1 & 797 & abbreviated & 0 & -0.11191 \\
\hline 4 & 3 & 11 & 1 & 1 & 734 & crackle & 0 & -0.28387 \\
\hline 5 & 3 & 12 & 1 & 1 & 954 & confiding & 0 & 0.316624 \\
\hline 6 & 3 & 13 & 1 & 1 & 810 & director's & 0 & -0.07643 \\
\hline 7 & 3 & 14 & 1 & 1 & 681 & appeals & 0 & -0.42853 \\
\hline 8 & 3 & 15 & 1 & 1 & 860 & postmaster's & 0 & 0.060049 \\
\hline 9 & 3 & 16 & 1 & 1 & 598 & videotape & 0 & -0.65508 \\
\hline 10 & 3 & 17 & 1 & 1 & 594 & plus & 0 & -0.666 \\
\hline 11 & 3 & 19 & 1 & 1 & 647 & incise & 0 & -0.52134 \\
\hline 12 & 3 & 20 & 1 & 1 & 752 & godchild & 0 & -0.23474 \\
\hline 13 & 3 & 23 & 1 & 1 & 804 & inspected & 0 & -0.0928 \\
\hline 14 & 3 & 25 & 1 & 1 & 666 & giggles & 0 & -0.46948 \\
\hline
\end{tabular}

Figure 17. Trial-level data for a typical participant. 
on behavioral data from the naming and lexical decision results, or a myriad of descriptive characteristics. As an example, one could easily generate a list of words that produce lexical decision latencies within the range of 800 $850 \mathrm{msec}$, are 12 letters in length, and have 5 orthographic neighbors. This database will also be important in the area of memory and text comprehension, wherein researchers investigate the effects of variables above and beyond differences in isolated lexical processing. For example, one could equate the lexical processing characteristics across different texts at the word level, as reflected by naming and lexical decision performance obtained from the ELP, to investigate the influence of higher-level integration processes. Finally, this data set could be useful in obtaining baselines across counterbalanced lists, which ultimately will reduce the error variance in standard factorial designs that counterbalance targets across conditions.

In summary, the ELP is potentially useful for equating items across a wide variety of research enterprises that use words as the primary stimulus. Obviously, for a number of reasons, the lexical characteristics included in the ELP are not, and were not meant to be, exhaustive. For example, metrics that reflect spelling-sound relationships and semantic characteristics are not included, and these measures, along with others, have been shown to be relevant in word recognition performance (see Balota et al., 2004 , for a review). Nevertheless, we believe the variables available represent a good start.

\section{Examples of ELP Applications in the Recent Literature}

Although the ELP has been on-line for a relatively short time, it has already been fruitfully employed in published papers by different researchers for disparate purposes. For example, in Balota et al.'s (2004) megastudy, the ability of different variables to predict naming and lexical decision latencies for 2,428 monosyllabic words was investigated. In order to test the replicability from this study, the same 2,428 words were extracted from the ELP. The results provided a remarkably clear replication of the predictive power of the same set of variables (see Balota et al., 2004, Figures 10 and 11). Of course, this also is relevant to the discussion above regarding the reliability of data from studies wherein participants produce responses to 2,000 to 3,000 words and/or nonwords. In addition, New, Ferrand, Pallier, and Brysbaert (2006) examined length effects using lexical decision latencies for 33,006 items from the ELP. They observed an interesting quadratic relationship between length and lexical decision performance, with facilitatory effects (faster latencies for longer words) for shorter words, null effects for medium-length words, and inhibitory effects (slower latencies for longer words) for longer words. This may explain why null length effects are typically observed in factorial lexical decision experiments, where medium-length stimuli are most often used. Finally, Kello (2006) has recently developed a junction model of word reading, which employs different modes (lexical and sublexical) of processing. The most interesting aspect of this model is that it can simulate response times for multisyllabic words. Kello has so far tested the model against 30,000 response times from the ELP. Intriguingly, the model was able to account for a substantial proportion of variance in both lexical decision and naming performance, after articulatory characteristics and length were partialed out. We anticipate that these initial published demonstrations of the utility of the ELP will markedly increase in the future, and we hope that the database will be helpful for researchers across diverse areas in cognitive science.

\section{AUTHOR NOTE}

This research was supported by Grant AGO3991 from the National Institute on Aging and by Grant BCS 0001801 from the National Science Foundation. Thanks are extended to Greg Burgess and Mark Law for programming and help in the early planning stages of the project, and to Christine Abadire, Melissa Davis, April Fugett, Leilani Goodman, Jeff Johnson, Lawrence Locker, Jr., Susan Marshall, Tameka Roberson, Jeff Templeton, and Mark Yates for help in various aspects of collecting the data. Thanks are also extended to Curt Burgess for providing the word frequency estimates from the HAL database, and Stephen Isard and Susan Fitt for allowing access to the Unisyn Lexicon for the phonological information available on the ELP website. Correspondence concerning this article should be addressed to D. A. Balota, Department of Psychology, Washington University, St. Louis, MO 63130 (e-mail: dbalota@artsci.wustl.edu).

\section{REFERENCES}

ANDREws, S. (1997). The effect of orthographic similarity on lexical retrieval: Resolving neighborhood conflicts. Psychonomic Bulletin \& Review, 4, 439-461.

Ans, B., Carbonnel, S., \& Valdois, S. (1998). A connectionist multitrace memory model of polysyllabic word reading. Psychological Review, 105, 678-723.

BaAyen, R. H., Piepenbrock, R., \& van Rijn, H. (1993). The CELEX lexical database. Philadelphia, PA: Linguistic Data Consortium, University of Pennsylvania.

Balota, D. A., \& Chumbley, J. I. (1984). Are lexical decisions a good measure of lexical access? The role of word frequency in the neglected decision stage. Journal of Experimental Psychology: Human Perception \& Performance, 10, 340-357.

Balota, D. A., \& Chumbley, J. I. (1985). The locus of word-frequency effects in the pronunciation task: Lexical access and/or production? Journal of Memory \& Language, 24, 89-106.

Balota, D. A., Cortese, M. J., Sergent-Marshall, S. D., Spieler, D. H., \& YAP, M. J. (2004). Visual word recognition of single-syllable words. Journal of Experimental Psychology: General, 133, 283-316.

Balota, D. A., \& Spieler, D. H. (1998). The utility of item-level analyses in model evaluation: A reply to Seidenberg and Plaut. Psychological Science, 9, 238-240.

Besner, D., \& Bourassa, D. C. (1995, June). Localist and parallel processing models of visual word recognition: A few more words. Paper presented at the Brain Behavior, and Cognitive Science Society, Halifax, CA.

Cattell, J. M. (1886). The time it takes to see and name objects. Mind, 11, 63-65.

Centre for Speech Technology Research, University of EdinBURGH. (n.d.). Unisyn lexicon [Data file]. Retrieved July 19, 2004, from www.cstr.ed.ac.uk/projects/unisyn/.

Chateau, D., \& Jared, D. (2003). Spelling-sound consistency effects in disyllabic word naming. Journal of Memory \& Language, 48, 255-280.

Coltheart, M., Davelaar, E., Jonasson, J., \& Besner, D. (1977). Access to the internal lexicon. In S. Dornic (Ed.), Attention and performance VI (pp. 535-555). Hillsdale, NJ: Erlbaum.

Coltheart, M., Rastle, K., Perry, C., Langdon, R., \& Ziegler, J. (2001). DRC: A dual route cascaded model of visual word recognition and reading aloud. Psychological Review, 108, 204-256.

CutLer, A. (1981). Making up materials is a confounded nuisance, or: Will we be able to run any psycholinguistic experiments at all in 1990 ? Cognition, 10, 65-70. 
Faust, M. E., Balota, D. A., Spieler, D. H., \& Ferraro, F. R. (1999). Individual differences in information-processing rate and amount: Implications for group differences in response latency. Psychological Bulletin, 125, 777-799.

FORSTER, K. I. (2000). The potential for experimenter bias effects in word recognition experiments. Memory \& Cognition, 28, 1109-1115.

Grainger, J., \& JACOBS, A. M. (1996). Orthographic processing in visual word recognition: A multiple read-out model. Psychological Review, 103, 518-565.

Horne, J. A., \& Ostberg, O. (1976). A self-assessment questionnaire to determine morningness-eveningness in human circadian rhythms. International Journal of Chronobiology, 4, 97-110.

Jared, D., McRae, K., \& SeidenberG, M. S. (1990). The basis of consistency effects in word naming. Journal of Memory \& Language, 29, 687-715.

Keefe, D. E., \& Neely, J. H. (1990). Semantic priming in the pronunciation task: The role of prospective prime-generated expectancies. Memory \& Cognition, 18, 289-298.

Kello, C. T. (2006). Considering the junction model of lexical processing. In S. Andrews (Ed.), From inkmarks to ideas: Current issues in lexical processing (pp. 50-75). New York: Psychology Press.

Kessler, B., Treiman, R., \& Mullennix, J. (2002). Phonetic biases in voice key response time measurements. Journal of Memory \& Language, 47, 145-171.

KuČERA, H., \& FranCIs, W. (1967). Computational analysis of presentday American English. Providence, RI: Brown University Press.

Lund, K., \& Burgess, C. (1996) Producing high-dimensional semantic spaces from lexical co-occurrence. Behavior Research Methods, Instruments, \& Computers, 28, 203-208.

McClelland, J. L., \& Rumelhart, D. E. (1981). An interactive activation model of context effects in letter perception: Part 1. An account of basic findings. Psychological Review, 88, 375-407.

NeELY, J. H. (1977). Semantic priming and retrieval from lexical memory: Roles of inhibitionless spreading activation and limited-capacity attention. Journal of Experimental Psychology: General, 106, 226-254.

Neely, J. H., Keefe, D. E., \& Ross, K. L. (1989). Semantic priming in the lexical decision task: Roles of prospective prime-generated expectancies and retrospective semantic matching. Journal of Experimental Psychology: Learning, Memory, \& Cognition, 15, 1003-1019.

New, B., Ferrand, L., Pallier, C., \& Brysbaert, M. (2006). Reex- amining word length effects in visual word recognition: New evidence from the English Lexicon Project. Psychonomic Bulletin \& Review, 13, 45-52.

Petersen, S. E., Fox, P. T., Posner, M. I., Mintun, M., \& Raichle, M. E. (1989). Positron emission tomographic studies of the processing of single words. Journal of Cognitive Neuroscience, 1, 153-170.

Plaut, D. C., McClelland, J. L., Seidenberg, M. S., \& Patterson, K. (1996). Understanding normal and impaired word reading: Computational principles in quasi-regular domains. Psychological Review, 103, 56-115.

Seidenberg, M. S., \& McClelland, J. L. (1989). A distributed developmental model of word recognition and naming. Psychological Review, 96, 523-568.

SeidenberG, M. S., \& Waters, G. S. (1989, November). Naming words aloud: A mega-study. Paper presented at the meeting of the Psychonomic Society, Atlanta, GA.

ShIPlEY, W. C. (1940). A self-administering scale for measuring intellectual impairment and deterioration. Journal of Psychology, 9, 371-377.

Spieler, D. H., \& BAlota, D. A. (1997). Bringing computational models of word naming down to the item level. Psychological Science, 8 , 411-416.

Stroop, J. R. (1935). Studies of interference in serial verbal reactions. Journal of Experimental Psychology, 18, 643-662.

Taraban, R., \& McClelland, J. L. (1987). Conspiracy effects in word pronunciation. Journal of Memory \& Language, 26, 608-631.

Treiman, R., Mullennix, J., Bijeljac-Babic, R., \& RichmondWelty, E. D. (1995). The special role of rimes in the description, use, and acquisition of English orthography. Journal of Experimental Psychology: General, 124, 107-136.

Zevin, J. D., \& SeIDEnberG, M. S. (2002). Age of acquisition effects in word reading and other tasks. Journal of Memory \& Language, 47, 1-29.

Zorzi, M., Houghton, G., \& Butterworth, B. (1998). Two routes or one in reading aloud? A connectionist dual-process model. Journal of Experimental Psychology: Human Perception \& Performance, 24, 1131-1161.

(Manuscript received February 3, 2006; revision accepted for publication April 26, 2006.) 